\title{
Pronóstico de la ventilación mecánica invasiva en el paciente obeso críticamente enfermo
}

\author{
Alejandro Montaño Jiménez, ${ }^{*}$ Claudia Ivette Olvera Guzmán, ${ }^{*}$ Janet Silvia Aguirre Sánchez, \\ Gilberto Camarena Alejo, ${ }^{*}$ Juvenal Franco Granillo*
}

\section{RESUMEN}

Introducción: La obesidad es una enfermedad crónica que se relaciona de manera causal con múltiples patologías y aumento de morbilidad y mortalidad. A nivel respiratorio genera disminución de distensibilidad y volumen pulmonar, con colapso de vías aéreas; esto, junto con la limitación del diafragma, favorece el colapso pulmonar y formación de atelectasias, con disminución de oxigenación y aumento en el riesgo de infección.

Métodos: Estudio retrospectivo, transversal, en pacientes con ventilación mecánica invasiva (VMI) por más de 48 horas. Se valoraron datos demográficos y de VM.

Resultados: Se analizaron 50 pacientes, en dos grupos: no obesos con IMC < $30 \mathrm{~kg} / \mathrm{m}^{2}$, y obesos con IMC $\geq 30 \mathrm{~kg} / \mathrm{m}^{2}$, con 35 y 15 pacientes respectivamente. Se encontró diferencia en la relación $\mathrm{PaO}_{2} / \mathrm{FiO}_{2}$ siendo mayor en no obesos $193 \pm 112(54-415)$ que en obesos $116 \pm 58(41-260) p<0.0001$. Se utilizó mayor PEEP (Presión positiva al final de la espiración) en los obesos $11 \pm 2.8$ (5-16) $\mathrm{cmH}_{2} \mathrm{O}$ que en los no obesos $9 \pm 2.4$ (5-16) $\mathrm{cmH}_{2} \mathrm{O} p<0.007$. La mortalidad fue de $28.6 \%(n=10)$ en no obesos y de $40 \%(n=6)$ en obesos; no presentó diferencia estadísticamente significativa. No se encontraron diferencias en los tiempos de ventilación mecánica y de estancia en UCl e intrahospitalaria. Análisis: En este estudio no se encontró diferencia significativa en mortalidad entre pacientes obesos y no obesos, a diferencia de lo que se ha encontrado en otros estudios; aunque la diferencia fue de $12 \%$, en el grupo de obesos, se encontró de manera inicial menor oxigenación y requirieron de mayor PEEP para ventilarse, recuperando la oxigenación a las 24 horas.

Conclusiones: No se encontró diferencia en este estudio. Se requiere mayor número de pacientes para valorar efecto en mortalidad.

Palabras clave: Ventilación mecánica invasiva, obesidad, sobrepeso.

\section{SUMMARY}

Introduction: Obesity has become a chronic illness which casually relates with multiple pathologies as well as with an increase in morbidity and mortality rates. On the respiratory system, it diminishes lung compliance, and tidal volume with airway collapse. This issue along with movement limitation from the diaphragm favors lung collapse, and atelectasis appearance causing oxygenation reduction while rising the risk of infection.

Methodology: Retrospective transversal assay performed on patients undergoing invasive mechanical ventilation for over 48 hours. Demographic and mechanical ventilation values are to be evaluated.

Results: 50 patients were analyzed having a tow group distribution, nonobese with a Body mass index (BMI) under $30 \mathrm{~kg} / \mathrm{m}^{2}$, and obese with a Body mass index (BMI) over $30 \mathrm{~kg} / \mathrm{m}^{2}$, having 35 and 15 patients respectively. A difference between the relation of the values $\mathrm{PaO}_{2} / \mathrm{FiO}_{2}$ was found been higher in the non-obese group $193 \pm 112$ (54-415), while in the obese group it was $116 \pm 58(41-260) p<0.0001$. A bigger value for the Positive end-expiratory pressure (PEEP) was used in obese patients $11 \pm 2.8$ (516) $\mathrm{CmH}_{2} \mathrm{O}$ compared to the non-obese group with which the PEEP value was $9 \pm 2.4$ (5-16) $\mathrm{cmH}_{2} \mathrm{O} p<0.007$. The mortality was of $28.6 \%(n=10)$ in the non-obese group while it was $40 \%(n=6)$ in the obese group, no relevant statistical differences were observed. There were no differences in the ventilation time values, neither was a difference for the hospital care or the Intensive Care Unit admission times.

Analysis: No significant difference was observed regarding mortality rates between obese and non-obese group, in contrast to other assays. While a $12 \%$ difference was found in the oxygenation values between the two groups, having the obese group a lower oxygenation value.

Conclusion: No significant differences were found in this study. A greater number of patients would be needed to evaluate the effect on the mortality rate. Key words: Invasive mechanical ventilation, obesity, overweight.

\section{* Centro Médico ABC, Ciudad de México, México.}

Recepción: 01/09/2017. Aceptación:

\section{RESUMO}

Introdução: A obesidade é uma doença crônica que está casualmente relacionada a múltiplas patologias e aumento da morbidade e mortalidade. $A$ nível respiratório, causa diminuição da complacência e do volume pulmonar com colapso das vias aéreas; isso, juntamente com a limitação do diafragma favorece o colapso dos pulmões e a formação de atelectasias com diminuição da oxigenação e aumento do risco de infecção.

Métodos: Estudo retrospectivo, transversal com ventilação mecânica invasiva (VMI) por mais de 48 horas. Se valoraron datos demográficos y de VM.

Resultados: Foram analisados 50 pacientes em 2 grupos: não obesos com $I M C<30 \mathrm{~kg} / \mathrm{m}^{2}$, e obesos com IMC $\geq 30 \mathrm{~kg} / \mathrm{m}^{2} \mathrm{com} 35$ e 15 pacientes respectivamente. Foi encontrada uma diferença na relação $\mathrm{PaO} 2 / \mathrm{FiO} 2$, sendo maior em não obesos $193 \pm 112$ (54-415) do que em obesos $116 \pm 58$ (41-260) $p<0.0001$. Utilizadou-se maior PEEP (pressão expiratória final positiva) nos obesos $11 \pm 2.8$ (5-16) cmH2O do que nos não obesos $9 \pm 2,4$ (5-16) $\mathrm{cmH}_{2} \mathrm{O}$ $p<0.007$. A mortalidade foi de $28.6 \%(n=10)$ em pacientes não-obesos e de $40 \%(n=6)$ em obesos, não apresentando diferença estatisticamente significante. Não foram encontradas diferenças no tempo de ventilação mecânica, permanência em UTI e hospitalar.

Análise: Neste estudo não foi encontrada diferença significativa na mortalidade entre pacientes obesos e não obesos, em contraste com outros estudos, embora a diferença tenha sido de $12 \%$, no grupo de pacientes obesos inicialmente encontrou-se eles menor oxigenação e necessitaram de maior PEEP para ventilar, recuperando a oxigenação às 24 horas.

Palavras-chave: Ventilação mecânica invasiva, obesidade, excesso de peso.

\section{INTRODUCCIÓN}

La obesidad es una enfermedad crónica que se ha convertido en un problema de salud pública; se relaciona de manera causal con múltiples patologías como diabetes y alteraciones cardiovasculares, así como con aumento de morbilidad y mortalidad. ${ }^{1}$

Este problema tiene mayor relevancia debido a la gran prevalencia que presenta y que ha ido en aumento en todo el mundo, según la Organización Mundial de la Salud. Hay que considerar también que el aumento en obesidad en los adultos, se acompaña con aumento de peso en niños y adolescentes, lo que los hace susceptibles de obesidad temprana y sus complicaciones. $^{2}$

En México, según la Encuesta Nacional de Salud y Nutrición 2016 (ENSANUT2016), la prevalencia combinada de obesidad y sobrepeso en adultos mayores de 20 años es del $72.5 \%$. En los últimos cuatro años se encontró un aumento de $1.3 \%$, el cual, aunque no es significativo estadísticamente, sí es relevante a nivel nacional. Al observar las estadísticas de los últimos 28 años, en mujeres de 20 a 49 años, el sobrepeso aumentó $42.9 \%$ y la prevalencia de obesidad en $290 \%$; en los últimos cuatro años, la obesidad se incrementó en $5.4 \%$. En el caso de los hombres, durante un periodo de 16 años, el sobrepeso aumentó $1.1 \%$ y la obesidad en $14.3 \% .^{3}$ 
La obesidad se define como una enfermedad en la cual el exceso de grasa corporal se ha acumulado de tal manera que puede generar efectos adversos a la salud. El incremento de la grasa corporal trae profundos cambios en la fisiología, la cual puede depender del tipo de obesidad: la obesidad alrededor del tórax y abdomen puede restringir la respiración; a nivel visceral-abdominal se asocia a hipertensión, resistencia a la insulina, diabetes y dislipidemia; la obesidad generalizada afecta el volumen sanguíneo y la función cardiovascular. ${ }^{1,2}$ Estos cambios también limitan la capacidad del cuerpo para adaptarse en situaciones de estrés, como sucede en el paciente críticamente enfermo y éstos pueden incluir: inflamación, estrés oxidativo, alteraciones en la función endotelial, adipocitocinas, entre otros, ${ }^{4}$ y aunque se predice peor pronóstico para los pacientes con obesidad, los resultados en los estudios han sido no concluyentes e incluso en muchas series se ha encontrado una disminución en mortalidad en este grupo de pacientes; esto ha sido llamado «paradoja de la obesidad» ya que el sobrepeso y la obesidad parecieran tener un factor protector en los pacientes críticamente enfermos, mientras que en los pacientes con bajo peso pareciera haber una mortalidad aumentada. ${ }^{5,6}$

En un metaanálisis, se observó que la mortalidad se presenta en forma de U. Los pacientes desnutridos (IMC menor de 18.5) y los pacientes con obesidad mórbida (IMC > 40) tienen una mayor mortalidad, mientras que el sobrepeso y la obesidad moderada parecieran tener un factor protector para mortalidad en el estado crítico.

En estos pacientes, la oxigenación se encuentra disminuida, mientras que, a nivel metabólico, existe un incremento en el trabajo respiratorio en reposo y el consumo de oxígeno se encuentra aumentado en 1.5 veces. Esto trae como consecuencia disminución de la oxigenación e hipercapnia que puede favorecer disminución del estímulo respiratorio, lo cual suele agravar las alteraciones de oxigenación y generar mayor hipercapnia. $^{2}$

Se ha observado una correlación entre el índice de masa corporal e hiperreactividad de la vía aérea; además se ha encontrado aumento de marcadores de inflamación en pacientes obesos, asociado a mayor hiperreactividad. Se han encontrado, también, mayores niveles de leptina, tanto en lavado bronquial como en sangre; éstos se asocian a inflamación y estrés oxidativo; su receptor se encuentra expresado en el epitelio respiratorio, favoreciendo la inflamación y obstrucción de la vía aérea. Estos cambios se ven reflejados, por ejemplo, en que el asma es más común en obesos, y que después de una cirugía bariátrica se consigue mejoría en el control de ésta.

A nivel pulmonar, el exceso de grasa en abdomen y tórax genera disminución de la distensibilidad pulmonar y de la pared torácica; el diafragma es desplazado de manera pasiva hacia el tórax, disminuyendo la capacidad pulmonar y la distensibilidad hasta 35\%, con una disminución del volumen pulmonar al final de la espiración y, por lo tanto, de la capacidad residual funcional, que junto con la hiperreactividad bronquial y la obstrucción de vías aéreas ocasiona el colapso de las mismas y la disminución del volumen pulmonar, favoreciendo nuevamente dicho colapso. En este contexto, el cierre repetido de vías aéreas favorece la inflamación, lo que puede reforzar la hiperreacción bronquial y obstrucción de las vías aéreas. ${ }^{7}$

El colapso de la vía aérea y la disminución de la capacidad pulmonar, junto con la limitación del diafragma, favorecen el colapso pulmonar y la formación de atelectasias; todos estos cambios repercuten en la oxigenación y ventilación de los pacientes obesos. ${ }^{8}$

A nivel cardiopulmonar, la hipoxemia crónica y la hipercapnia generan hipertensión arterial pulmonar, con mayor deterioro de la oxigenación y con deterioro de la función cardiaca. Además, en los pacientes obesos se presenta aumento del volumen circulante y del consumo de oxígeno, lo que provoca que se requiera mayor gasto cardiaco. Existe también aumento de las resistencias periféricas, que junto con el aumento del volumen circulante provocan mayor postcarga, generando hipertrofia concéntrica del ventrículo izquierdo, llevando al paciente a falla cardiaca, tanto sistólica como diastólica. ${ }^{2}$

Estos cambios en la fisiología respiratoria hacen que existan diferencias en la ventilación mecánica, ya que se tiene disminución de la capacidad funcional residual, aumento de resistencias, disminución de distensibilidad y tendencia al colapso de las vías aéreas. Otro punto a considerar, es que se tienden a sobrestimar las necesidades del volumen corriente al considerar el peso real. Al revisar los volúmenes utilizados en pacientes con diferentes grados de IMC, Anzueto y cols. reportaron el uso de volúmenes mayores de $10 \mathrm{~mL} / \mathrm{kg}$ de peso predicho en los pacientes con IMC mayor de $30 \mathrm{~kg} / \mathrm{m}^{2} .9$

Como se comentó previamente, los pacientes obesos presentan una «paradoja», ya que parecen tener una menor mortalidad a pesar de que de manera teórica se encuentran en una desventaja fisiológica. Durante la ventilación mecánica se repite este patrón. En un metaanálisis de 24 estudios, todos con pacientes con síndrome de distrés respiratorio agudo, se encontró menor mortalidad con aumento de IMC, este efecto es mayor en el grupo de obesidad (IMC $30-39.9 \mathrm{~kg} / \mathrm{m}^{2}$ ) y se ve disminuido en el grupo de obesidad mórbida. En cuanto a la morbilidad, se encontraron mayores complicaciones en los pacientes con IMC mayor de $30 \mathrm{~kg} / \mathrm{m}^{2}$. ${ }^{6,10}$ 


\section{MATERIAL Y MÉTODOS}

Se realizó un estudio retrospectivo transversal en pacientes con ventilación mecánica invasiva en una Unidad de Terapia Intensiva Mixta. Desde el primero de marzo de 2016 al 28 de febrero de 2017.

Se revisaron los expedientes de pacientes egresados de la Unidad de Cuidados Intensivos, seleccionando aquellos que presentaron ventilación mecánica invasiva por más de 48 horas, por cualquier causa.

Criterios de selección: Criterios de inclusión: $\mathrm{Pa}$ cientes de 18 años o mayores que fueron tratados con ventilación mecánica invasiva por más de 48 horas.

Criterios de exclusión: Pacientes que fueran trasladados a otra institución, expediente incompleto, pacientes sin monitoreo con gasometrías arteriales y pacientes con trámite de voluntad anticipada.

\section{Análisis}

De los pacientes incluidos, se realizó búsqueda en expediente electrónico tanto TIMSA como OnBase. Se recopilaron datos demográficos y clínicos, así como programación del ventilador y se registraron en una base de datos electrónica en Microsoft Excel. Los datos demográficos fueron edad, sexo, peso y talla; se calculó IMC con la fórmula IMC = peso en kg/talla en metros al cuadrado, tiempo de ventilación mecánica, días de estancia en la UCI, días de estancia intrahospitalaria y mortalidad al egreso. Se calcularon puntajes de SOFA y APACHE II; se recabaron datos gasométricos y de parámetros de ventilación mecánica al ingreso y a las 48 horas.

Se realizó análisis estadístico con SPSS 22 y un análisis descriptivo; para el análisis comparativo se utilizó Chi cuadrada $\left(\chi^{2}\right)$ para las variables dicotómicas y $t$ de Student para las variables continuas.

\section{RESULTADOS}

Se analizaron 50 pacientes, con una edad promedio de $64 \pm 17$ (20-89) años, de ellos 31 (62\%) son hombres y $38 \%$ mujeres. La media de peso fue de $81 \mathrm{~kg} \pm 22$, con un rango de 50 a 145, con un IMC promedio de $28.8 \pm$ $7.0(18.4-51.4) \mathrm{kg} / \mathrm{m}^{2}$; el menor fue $18.4 \mathrm{~kg} / \mathrm{m}^{2}$ y el mayor $51.5 \mathrm{~kg} / \mathrm{m}$. Solamente hubo un paciente con peso bajo, $16(32 \%)$ pacientes tenían peso normal, 18 (36\%) presentaron sobrepeso y 15 (30\%) obesidad, de los cuales cinco (10\%) tuvieron obesidad mórbida. Entre las comorbilidades que presentaron, 12 (24\%) pacientes tenían diabetes mellitus, la mayor parte de ellos en los grupos de sobrepeso y obesidad; nueve (18\%) tenían EPOC, nueve $(18 \%)$ insuficiencia cardiaca y un paciente fibrosis pulmonar. En la Tabla 1 se muestran las características generales de los pacientes y de cada grupo de IMC.
Los principales diagnósticos de ingreso fueron: 12 pacientes (24\%) con neumonía, siete (14\%) con sepsis abdominal, cuatro $(28 \%)$ con infección de tejidos blandos, tres $(6 \%)$ por estado postparo, tres $(6 \%)$ por traumatismo craneoencefálico (TCE) y tres $(6 \%)$ con enfermedad pulmonar obstructiva crónica (EPOC). Al momento del inicio de la ventilación mecánica el promedio de puntaje de SOFA fue de $8 \pm 2.8$ (3-15), el APACHE II promedio fue de $16 \pm 2.8$ (3-41. Las principales causas de inicio de la ventilación mecánica fueron hipoxemia en 20 (40\%), hipercapnia en 11 (22\%), ocho $(16 \%)$ ingresaron en postoperatorio y seis $(12 \%)$ por falla cardiaca. Las principales modalidades de ventilación utilizadas fueron: asisto control volumen (ACV) en 29 (58\%), asisto control presión (ACP) en $10(20 \%)$, Bilevel en cinco (10\%), ventilación por liberación de presión (APRV, por sus siglas en inglés) en dos (4\%) y ventilación presión soporte (VPS) en dos (4\%). Al inicio de la ventilación, el volumen corriente promedio fue de 400 $\pm 64 \mathrm{~mL}$ (230-580), que corresponden en promedio a $6.4 \mathrm{~mL} / \mathrm{kg}$ de peso predicho +0.9 (3.7-9.1) con PEEP promedio de $9 \pm 2.6 \mathrm{cmH}_{2} \mathrm{O} \pm 2.6 .^{5-6}$ El promedio de peso predicho fue de $62 \pm 8.9(45-81) \mathrm{kg}$. Presentaron una relación $\mathrm{PaO}_{2} / \mathrm{FiO}_{2}$ de $169 \pm 104(41-415)$ y una distensibilidad promedio de $35 \pm 13(10-74) \mathrm{mL} / \mathrm{cmH}_{2} \mathrm{O}$.

A las 48 horas, los pacientes tuvieron relación $\mathrm{PaO}_{2} /$ $\mathrm{FiO}_{2}$ de $248 \pm 93$ (87-454), con distensibilidad de $39 \pm$ $14.2 \mathrm{~mL} / \mathrm{cmH}_{2} \mathrm{O}$ (13-76); esto representa un aumento en promedio de 62 en la relación $\mathrm{PaO}_{2} / \mathrm{FiO}_{2}$. El volumen corriente promedio a las 48 horas fue de $435 \pm$ $90 \mathrm{~mL}$ (270-750), que corresponden en promedio a 6.9 (4.6-10.5) $\mathrm{mL} / \mathrm{kg}$ de peso predicho, con PEEP promedio de $9 \pm 2.5$ (5-16) $\mathrm{cmH}_{2} \mathrm{O}$ y con una media de $8 \pm 7$ (239) días de ventilación mecánica.

La mortalidad global fue de $32 \%$, la estancia media en la UCl fue de $15 \pm 11$ (2-69), con una estancia intrahospitalaria promedio de $20 \pm 19$ (2-124) días. En siete (14\%) pacientes se utilizó ventilación en posición prono y en cinco (10\%) pacientes se realizó traqueostomía.

Posteriormente se dividieron los pacientes en dos grupos: pacientes no obesos con IMC $<30 \mathrm{~kg} / \mathrm{m}^{2}$, y pacientes obesos con IMC $\geq 30 \mathrm{~kg} / \mathrm{m}^{2}$, los cuales tuvieron 35 y 15 pacientes respectivamente. En el grupo de los no obesos, la edad promedio fue de $65 \pm 16(20-88)$ años, de éstos 23 (65.7\%) eran hombres y 12 (36.3\%) mujeres. En el grupo de obesos la edad promedio fue de $63 \pm 19$ (30-89) años y ocho (53\%) eran hombres mientras que siete (47\%) eran mujeres. No se observó diferencia estadísticamente significativa ni en edad ni en género.

Al comparar los puntajes de gravedad, no se encontró diferencia significativa entre ambos grupos para SOFA: $9 \pm 2.9$ (3-15) para no obesos y $8 \pm 2.3$ (3-13) para obesos y APACHE II: $17 \pm 8$ (3-41) de no obesos y $16 \pm 5$ (8-17) para obesos. Entre las comorbilidades 
solamente se encontró diferencia con respecto a diabetes mellitus, la cual se presentó en cinco (14.4\%) en el grupo de no obesos y siete (46.7\%) en el grupo de obe$\operatorname{sos} p<0.0001$; en cuanto a EPOC, fibrosis pulmonar y falla cardiaca no hubo diferencia significativa. En los diagnósticos de ingreso, la sepsis fue más común en el grupo de no obesos con 10 (28\%) que en el de obesos con dos $(13 \%) p<0.02$; en el resto de los diagnósticos no se encontró diferencia estadística o no hubo suficientes pacientes para realizar análisis. En cuanto a la causa de inicio de ventilación mecánica invasiva, fue más común la hipoxemia en el grupo de obesos al compararlo con el de no obesos: ocho (53\%) y $13(34 \%)$ respectivamente, $p<0.01$, y también fue más común la falla cardiaca en este grupo: tres $(20 \%)$ versus tres $(8.6 \%)$ de los no obesos, $p<0.04$; el resto de los moti-

Tabla 1: Características demográficas de los pacientes.

\begin{tabular}{|c|c|c|c|c|c|c|}
\hline Total $n=50$ & & $\begin{array}{l}\text { Peso bajo } \\
n=1(2 \%)^{\star}\end{array}$ & $\begin{array}{l}\text { Peso normal } \\
n=16(32 \%)\end{array}$ & $\begin{array}{c}\text { Sobrepeso } \\
n=18(36 \%)\end{array}$ & $\begin{array}{c}\text { Obesidad } \\
n=10(20 \%)\end{array}$ & $\begin{array}{c}\text { Obesidad Mórbida } \\
n=5(10 \%)\end{array}$ \\
\hline Edad (años) & $64 \pm 17(20-89)$ & 79 & $61.0 \pm 19.4(20-86)$ & $68.0 \pm 14.8(42-88)$ & $70.0 \pm 18.3(34-89)$ & $50.0 \pm 14.6(30-67)$ \\
\hline Peso (real en kg) & $81 \pm 22(50-145)$ & 50 & $60.0 \pm 5.3(50-70)$ & $81.0 \pm 9.4(67-96)$ & $92.0 \pm 14.2(80-120)$ & $128.0 \pm 14.9(108-145)$ \\
\hline IMC $\left(\mathrm{kg} / \mathrm{m}^{2}\right)$ & $28.8 \pm 7.0(18.4-51.4)$ & 18.3 & $22.7 \pm 1.5(19-24)$ & $27.5 \pm 1.1(26-29)$ & $33.8 \pm 2.6(30-38)$ & $44.8 \pm 4.5(41-51)$ \\
\hline \multicolumn{7}{|l|}{ Género } \\
\hline Hombre & $\mathrm{n}=31(62 \%)$ & $n=1(100 \%)$ & $n=8(50 \%)$ & $\mathrm{n}=14(77.8 \%)$ & $n=5(50 \%)$ & $n=3(60 \%)$ \\
\hline Mujer & $n=19(38 \%)$ & - & $n=8(50 \%)$ & $\mathrm{n}=4(22.2 \%)$ & $\mathrm{n}=5(50 \%)$ & $n=2(40 \%)$ \\
\hline \multicolumn{7}{|l|}{ Comorbilidades } \\
\hline Diabetes & $n=12(24 \%)$ & - & $n=1(6.3 \%)$ & $n=4(22.2 \%)$ & $n=5(50 \%)$ & $n=2(40 \%)$ \\
\hline EPOC & $n=9(18 \%)$ & - & $n=3(18.8 \%)$ & $n=3(16.7 \%)$ & $n=3(30 \%)$ & - \\
\hline Fibrosis pulmonar & $n=1(2 \%)$ & - & $n=1(6.3 \%)$ & - & - & - \\
\hline Insuficiencia cardiaca & $\mathrm{n}=9(18 \%)$ & - & $\mathrm{n}=3(30 \%)$ & $\mathrm{n}=5(27.8 \%)$ & $\mathrm{n}=3(30 \%)$ & - \\
\hline SOFA & $8.0 \pm 2.8(3-15)$ & 3 & $9.0 \pm 2.6(4-13)$ & $9.0 \pm 2.9(6-15)$ & $8.0 \pm 1.5(6-11)$ & $7.0 \pm 3.6(3-13)$ \\
\hline APACHE II & $16.0 \pm 2.8(3-41)$ & 10 & $15.0 \pm 6.2(3-24)$ & $20.0 \pm 8.9(7-41)$ & $15.0 \pm 3.3(9-20)$ & $18.0 \pm 8.6(8-27)$ \\
\hline \multicolumn{7}{|l|}{ Diagnóstico de ingreso } \\
\hline Neumonía & $n=17(34 \%)$ & - & $n=7(43 \%)$ & $n=4(22.2 \%)$ & $n=4(40 \%)$ & $n=2(40 \%)$ \\
\hline Sepsis & $n=12(24 \%)$ & $n=1(100 \%)$ & $\mathrm{n}=4(25 \%)$ & $n=5(27.8 \%)$ & $n=1(10 \%)$ & $n=1(20 \%)$ \\
\hline Neurológico & $n=5(10 \%)$ & - & $n=4(25 \%)$ & $n=1(5.6 \%)$ & - & - \\
\hline Estado postparo & $n=3(6 \%)$ & - & $n=1(6.3 \%)$ & $n=2(11.1 \%)$ & - & - \\
\hline EPOC & $n=3(6 \%)$ & - & - & - & $n=3(30 \%)$ & - \\
\hline Derrame pleural & $\mathrm{n}=3(6 \%)$ & - & - & $n=3(16.7 \%)$ & - & - \\
\hline TEP & $\mathrm{n}=2(4 \%)$ & - & - & - & - & $\mathrm{n}=2(40 \%)$ \\
\hline Otros & $n=5(10 \%)$ & - & - & $n=3(16.7 \%)$ & $n=2(20 \%)$ & - \\
\hline \multicolumn{7}{|c|}{ Causa de inicio de ventilación mecánica } \\
\hline Hipoxemia & $n=20(40 \%)$ & - & $n=7(43.8 \%)$ & $n=5(27.8 \%)$ & $n=5(50 \%)$ & $\mathrm{n}=3(60 \%)$ \\
\hline Hipercapnia & $\mathrm{n}=11(22 \%)$ & - & $n=3(18.8 \%)$ & $n=4(22.2 \%)$ & $n=2(20 \%)$ & $n=2(40 \%)$ \\
\hline Postoperatorio & $n=8(16 \%)$ & - & $n=3(18.8 \%)$ & $n=3(16.7 \%)$ & $n=1(10 \%)$ & $n=1(20 \%)$ \\
\hline Falla cardiaca & $n=6(16 \%)$ & - & $n=1(6.3 \%)$ & $\mathrm{n}=2(11.1 \%)$ & $n=2(20 \%)$ & $n=1(20 \%)$ \\
\hline Trabajo respiratorio & $\mathrm{n}=4(8 \%)$ & - & - & $\mathrm{n}=2(11.1 \%)$ & $n=2(20 \%)$ & - \\
\hline Protección de vía aérea & $n=3(6 \%)$ & - & $n=2(12.5 \%)$ & $n=1(5.6 \%)$ & - & - \\
\hline Inestabilidad hemodinámica & $\mathrm{n}=3(6 \%)$ & $n=1(100 \%)$ & $n=1(6.3 \%)$ & $n=1(5.6 \%)$ & - & - \\
\hline Días de ventilación & $8 \pm 7(2-39)$ & 5 & $7.0 \pm 3.5(2-14)$ & $11.0 \pm 10.4(3-39)$ & $8.0 \pm 3.5(4-15)$ & $7.0 \pm 4.6(2-12)$ \\
\hline Estancia en la UCI (días) & $15 \pm 11(2-69)$ & 6 & $13.0 \pm 6.8(4-27)$ & $19.0 \pm 15.3(2-69)$ & $17.0 \pm 9.5(6-34)$ & $16.0 \pm 14(2-38)$ \\
\hline Estancia hospitalaria (días) & $20 \pm 19(2-124)$ & 6 & $16.0 \pm 9.5(4-41)$ & $20.0 \pm 15.4(2-69)$ & $19.0 \pm 8.7(6-34)$ & $39 \pm 50(2-124)$ \\
\hline \multicolumn{7}{|l|}{ Parámetros ventilatorios iniciales } \\
\hline $\mathrm{PaO}_{2} / \mathrm{FiO}_{2}$ & $169 \pm 104(41-415)$ & 110 & $176 \pm 117(60-415)$ & $212 \pm 109(54-387)$ & $127 \pm 62(68-260)$ & $96 \pm 50(41-173)$ \\
\hline PEEP $\left(\mathrm{cmH}_{2} \mathrm{O}\right)$ & $9.0 \pm 2.6(5-16)$ & 12 & $8.0 \pm 2.1(6-13)$ & $9.0 \pm 2.5(5-16)$ & $11.0 \pm 2.9(6-16)$ & $11.0 \pm 2.9(6-13)$ \\
\hline Volumen corriente $(\mathrm{mL})$ & $400 \pm 64(230-580)$ & 130 & $374.0 \pm 38.6(300-460)$ & $418 \pm 64(330-580)$ & $407 \pm 65(300-500)$ & $440 \pm 70(350-520)$ \\
\hline $\mathrm{mL} / \mathrm{kg}$ (predicho) & $6.4 \pm 0.9(3.7-9.1)$ & 3.7 & $6.3 \pm 0.7(4-8)$ & $6.4 \pm 0.7(5-8)$ & $6.7 \pm 0.7(6-8)$ & $7.0 \pm 1.3(6-9)$ \\
\hline Distensibilidad $\left(\mathrm{mL} / \mathrm{cmH}_{2} \mathrm{O}\right)$ & $35 \pm 13(10-74)$ & 26 & $38 \pm 13(10-58)$ & $37 \pm 14(15-74)$ & $34 \pm 9(21-49)$ & $28 \pm 11(27-45)$ \\
\hline Poder de distensión (j) & $11.4 \pm 5.1(2.7-24.5)$ & 8.2 & $9.4 \pm 4.8(2.7-20)$ & $12.1 \pm 4.3(6.4-24)$ & $12.0 \pm 5.9(4-22)$ & $14.0 \pm 5.6(9.2-24)$ \\
\hline \multicolumn{7}{|c|}{ Parámetros ventilatorios a las 48 horas } \\
\hline $\mathrm{PaO}_{2} / \mathrm{FiO}_{2}$ & $248 \pm 93(87-454)$ & 300 & $230 \pm 103(87-454)$ & $281 \pm 93(104-427)$ & $219 \pm 88(110-335)$ & $239 \pm 51(195-310)$ \\
\hline PEEP $\left(\mathrm{cmH}_{2} \mathrm{O}\right)$ & $9.0 \pm 2.5(5-16)$ & 12 & $8.0 \pm 2.5(5-14)$ & $9.0 \pm 1.9(6-14)$ & $10.0 \pm 2.4(8-16)$ & $12.0 \pm 3.1(8-15)$ \\
\hline Volumen corriente $(\mathrm{mL})$ & $435 \pm 90(270-750)$ & 540 & $402 \pm 51(350-530)$ & $419 \pm 50(320-500)$ & $467 \pm 128(320-650)$ & $494 \pm 172(270-750)$ \\
\hline mL/kg (predicho) & $6.9 \pm 1.3(4.6-10.5)$ & 8.8 & $6.7 \pm 1.0(5.7-10)$ & $6.4 \pm 0.7(5.8-8.1)$ & $7.3 \pm 1.6(5.3-10)$ & $7.8 \pm 2.5(4.6-10)$ \\
\hline Distensibilidad $\left(\mathrm{mL} / \mathrm{cmH}_{2} \mathrm{O}\right)$ & $39.0 \pm 14.2(13-76)$ & 61 & $41.0 \pm 15.5(13-76)$ & $42.0 \pm 12.1(21-64)$ & $39.0 \pm 16.2(20-69)$ & $37.0 \pm 14.4(21-49)$ \\
\hline Uso de prono & $\mathrm{n}=7(14 \%)$ & - & $n=3(18.8 \%)$ & $n=1(5.6 \%)$ & $n=2(20 \%)$ & $n=1(20 \%)$ \\
\hline Uso de traqueostomía & $n=5(10 \%)$ & - & $n=1(6.3 \%)$ & $n=3(16.7 \%)$ & - & $n=1(20 \%)$ \\
\hline Mortalidad & $n=16(32 \%)$ & - & $n=5(31.3 \%)$ & $n=5(27.8 \%)$ & $\mathrm{n}=4(40 \%)$ & $n=2(40 \%)$ \\
\hline
\end{tabular}

\footnotetext{
* No se realizó análisis estadístico ya que sólo hay un paciente en este grupo.
} 
vos de inicio de ventilación mecánica invasiva no fueron significativos; en la Tabla 2, se observan los resultados.

Al valorar la ventilación mecánica invasiva, se encontró diferencia en la relación $\mathrm{PaO}_{2} / \mathrm{FiO}_{2}$ siendo mayor en el grupo de no obesos $193 \pm 112$ (54-415) que en el grupo de obesos $116 \pm 58(41-260) p<0.0001$. Esta diferencia, después de 48 horas de ventilación mecáni-

Tabla 2: Grupos según peso

\begin{tabular}{|c|c|c|c|}
\hline & No obesos $(n=35)$ & Obesos $(n=15)$ & $p$ \\
\hline Edad (años) & $65 \pm 16(20-88)$ & $63 \pm 19(30-89)$ & NS \\
\hline Peso kg & $71 \pm 12.9(50.96)$ & $104 \pm 22.6(80-145)$ & $<0.0001$ \\
\hline \multicolumn{4}{|l|}{ Género } \\
\hline Hombre & $\mathrm{n}=23(65.7 \%)$ & $n=8(53 \%)$ & \multirow{2}{*}{ NS } \\
\hline Mujer & $\mathrm{n}=12(34.3 \%)$ & $\mathrm{n}=7(47 \%)$ & \\
\hline SOFA & $9 \pm 2.9(3-15)$ & $8 \pm 2.3(3-13)$ & NS \\
\hline APACHE II & $17 \pm 8(3-41)$ & $16 \pm 5(8-17)$ & NS \\
\hline \multicolumn{4}{|l|}{ Comorbilidades } \\
\hline Diabetes & $n=5(14.4 \%)$ & $\mathrm{n}=7(46.7 \%)$ & $<0.0001$ \\
\hline EPOC & $\mathrm{n}=6(17.1 \%)$ & $n=3(20 \%)$ & NS \\
\hline Fibrosis pulmonar & $n=1(2.9 \%)$ & $n=0$ & NS \\
\hline Falla cardiaca & $\mathrm{n}=6(17.1 \%)$ & $n=3(20 \%)$ & NS \\
\hline \multicolumn{4}{|l|}{ Diagnóstico de ingreso } \\
\hline Neumonía & $\mathrm{n}=11(31 \%)$ & $n=6(40 \%)$ & NS \\
\hline Sepsis & $\mathrm{n}=10(28 \%)$ & $n=2(13 \%)$ & $<0.02$ \\
\hline Neurológico & $n=5(14 \%)$ & - & $-\star *$ \\
\hline Estado postparo & $\mathrm{n}=3(8.6 \%)$ & - & $-* \star$ \\
\hline EPOC & - & $n=3(20 \%)$ & $-\star *$ \\
\hline Derrame pleural & $n=3(8.6 \%)$ & - & $-\star *$ \\
\hline TEP & - & $n=2(13 \%)$ & $-\star \star *$ \\
\hline Otros & $n=3(8.6 \%)$ & $n=2(13 \%)$ & NS \\
\hline \multicolumn{4}{|l|}{$\begin{array}{l}\text { Causa de inicio } \\
\text { de ventilación mecánica }\end{array}$} \\
\hline Hipoxemia & $n=13(34 \%)$ & $\mathrm{n}=8(53 \%)$ & $<0.01$ \\
\hline Hipercapnia & $\mathrm{n}=7(20 \%)$ & $\mathrm{n}=4(26 \%)$ & NS \\
\hline Postoperatorio & $\mathrm{n}=6(17.1 \%)$ & $n=2(13 \%)$ & NS \\
\hline Falla cardiaca & $n=3(8.6 \%)$ & $n=3(20 \%)$ & $<0.04$ \\
\hline Trabajo respiratorio & $n=2(5.7 \%)$ & $n=2(13 \%)$ & NS \\
\hline Protección de vía aérea & $n=3(6.8 \%)$ & - & $--^{* *}$ \\
\hline Inestabilidad hemodinámica & $n=3(8 \%)$ & - & $-\star *$ \\
\hline Días de ventilación & $9 \pm 8(2-39)$ & $7 \pm 3.8(2-15)$ & NS \\
\hline Estancia en la UCI (días) & $15 \pm 12(2-69)$ & $17 \pm 10(2-38)$ & NS \\
\hline Estancia hospitalaria (días) & $18 \pm 13(2-69)$ & $25 \pm 30(2-124)$ & NS \\
\hline \multicolumn{4}{|l|}{$\begin{array}{l}\text { Parámetros ventilatorios } \\
\text { iniciales }\end{array}$} \\
\hline $\mathrm{PaO}_{2} / \mathrm{FiO} 2$ & $193 \pm 112(54-415)$ & $116 \pm 58(41-260)$ & $<0.0001$ \\
\hline PEEP $\left(\mathrm{cmH}_{2} \mathrm{O}\right)$ & $9.0 \pm 2.4(5-16)$ & $11.0 \pm 2.8(5-16)$ & $<0.007$ \\
\hline Volumen corriente (mL) & $392 \pm 63(239-580)$ & $418 \pm 66(300-520)$ & NS \\
\hline mL/kg (predicho) & $6.3 \pm 0.8(3.7-8)$ & $6.8 \pm 0.9(5.6-9)$ & $<0.05$ \\
\hline Distensibilidad $\left(\mathrm{mL} / \mathrm{cmH}_{2} \mathrm{O}\right)$ & $37 \pm 14(10-74)$ & $32.0 \pm 9.7(17-49)$ & $\mathrm{NS}^{*}$ \\
\hline Poder de distensión (j) & $11.1 \pm 5.1(2.7-24)$ & $12.8 \pm 5.9(4-24)$ & NS \\
\hline \multicolumn{4}{|l|}{$\begin{array}{l}\text { Parámetros ventilatorios } \\
\text { a las } 48 \text { horas }\end{array}$} \\
\hline $\mathrm{PaO}_{2} / \mathrm{FiO}_{2}$ & $258 \pm 99(87-454)$ & $225 \pm 76(110-335)$ & NS \\
\hline PEEP $\left(\mathrm{cmH}_{2} \mathrm{O}\right)$ & $9.0 \pm 2.2(5-14)$ & $11.0 \pm 2.6(8-16)$ & $<0.007$ \\
\hline Volumen corriente (mL) & $414 \pm 54(320-540)$ & $476 \pm 139(270-750)$ & $<0.02$ \\
\hline mL/kg (predicho) & $6.6 \pm 0.9(5.7-10)$ & $7.5 \pm 1.9(4.6-10)$ & $<0.02$ \\
\hline Distensibilidad $\left(\mathrm{mL} \mathrm{cmH}_{2} \mathrm{O}\right)$ & $42 \pm 13(13-76)$ & $38 \pm 15(20-69)$ & NS \\
\hline Uso de prono & $\mathrm{n}=4(11.4 \%)$ & $n=3(20 \%)$ & NS \\
\hline Realización de traqueostomía & $\mathrm{n}=4(11.4 \%)$ & $n=1(6.7 \%)$ & NS \\
\hline Mortalidad & $n=10(28.6 \%)$ & $n=6(40 \%)$ & NS \\
\hline
\end{tabular}

*Diferencia de $14 \%,{ }^{* *}$ No se puede obtener valor estadístico. ca invasiva ya no es estadísticamente significativa con una relación $\mathrm{PaO}_{2} / \mathrm{FiO}_{2}$ de $258 \pm 99$ (87-454) y $225 \pm$ 76 (110-335) respectivamente. Se utilizó mayor PEEP en el grupo de pacientes obesos $11 \pm 2.8$ (5-16) $\mathrm{cmH}_{2} \mathrm{O}$ que en el grupo de los no obesos $9 \pm 2.4(5-16) \mathrm{cmH}_{2} \mathrm{O}$ $p<0.007 y$ se mantuvo la diferencia después de 48 horas, con PEEP de $9 \pm 2.2(5-14) \mathrm{cmH}_{2} \mathrm{O}$ en el grupo de no obesos y $11 \pm 2.6(8-16) \mathrm{cmH}_{2} \mathrm{O}(\mathrm{p}<0.007)$. El volumen corriente no mostró diferencia significativa al inicio de la ventilación mecánica invasiva: $392 \pm 63$ (239-580) $\mathrm{mL}$ en el grupo de no obesos y $418 \pm 66(300-520) \mathrm{mL}$ en obesos, pero al analizarlo calculado por peso predicho, se utilizó un volumen mayor en el grupo de obesos con diferencia estadística $p<0.05: 6.3 \pm 0.8(3.7-8) \mathrm{mL} /$ $\mathrm{kg}$ en no obesos y $6.8 \pm 0.9$ (5.6-9) $\mathrm{mL} / \mathrm{kg}$ en obesos. A las 48 horas, se continuó utilizando mayor volumen corriente en el grupo de obesos, esta vez siendo estadísticamente significativa la diferencia en volumen corriente $414 \pm 54$ (320-540) $\mathrm{mL}$ que corresponde a 6.6 $\pm 0.9(5.7-10) \mathrm{mL} / \mathrm{kg}$ de peso predicho en el grupo de no obesos comparado con $476 \pm 139$ (270-750) mL a $7.5+1.9(4.6-10) \mathrm{mL} / \mathrm{kg}$ de peso predicho en el grupo de obesos $p<0.02$. La distensibilidad fue menor en el grupo de los pacientes obesos $38 \pm 15(20-69) \mathrm{mL} /$ $\mathrm{cmH}_{2} \mathrm{O}$, versus $42 \pm 13(13-76) \mathrm{mL} / \mathrm{cmH}_{2} \mathrm{O}$ en los no obesos, aunque esta diferencia no fue estadísticamente significativa. Hay que considerar que la diferencia entre ambos grupos es de $14 \%$; a las 48 horas, la distensibilidad del grupo de no obesos aumentó a $42 \pm 13$ (13-76) $\mathrm{mL} / \mathrm{cmH}_{2} \mathrm{O}$, en el grupo de obesos aumentó a $38 \pm 15(20-69) \mathrm{mL} / \mathrm{cmH}_{2} \mathrm{O}$ y la diferencia entre ambas distensibilidades continuó siendo estadísticamente no significativa.

La duración de la ventilación mecánica invasiva no fue diferente entre ambos grupos, $9 \pm 8$ (2-39) días en el grupo de no obesos y $7 \pm 3.8(2-15)$ días en obesos. En cuanto a la estancia intrahospitalaria, el grupo de no obesos tuvo una estancia en UCl de $15 \pm 12$ (2-69) días y hospitalaria de $18 \pm 13$ (2-69) días; en el grupo de obesos la estancia en UCl fue de $17 \pm 10$ (2-38) días y la hospitalaria de $25 \pm 30$ (2-124) días, sin diferencia estadística entre ambos grupos. La mortalidad fue de $28.6 \%$ ( $n=10)$ en el grupo de no obesos y de $40 \%(n=6)$ en el grupo de obesos. Este resultado no presentó diferencia estadísticamente significativa; sin embargo se observa que la mortalidad es mayor cuantitativamente en el grupo de pacientes obesos mórbidos que la mortalidad en el grupo de pacientes obesos. Tampoco se encontró diferencia en la realización de traqueostomía, la cual se realizó en cuatro (11.4\%) pacientes en el grupo de no obesos y en un (6.7\%) paciente en el grupo de obesos. La posición prona se utilizó en cuatro $(11.4 \%)$ pacientes del grupo de no obesos y tres (20\%) pacientes en el grupo de obesos, lo cual no fue estadísticamente significativo. 


\section{DISCUSIÓN}

Se analizaron 50 pacientes cuya media de peso fue de $81 \mathrm{~kg}$ y de IMC de $28.8 \mathrm{~kg} / \mathrm{m}^{2}$, lo que los ubica dentro de la categoría de sobrepeso. La distribución dentro de los diferentes grupos dependiendo del IMC fue homogénea, excepto en el caso del grupo de bajo peso, el cual tuvo solamente un paciente. Esta distribución de los grupos refleja la población del hospital, el cual se encuentra en una zona urbana y es de asistencia privada, por lo que se trata de población que ya ha presentado transición económica y pertenece a un área industrializada. Esto provoca que el sobrepeso reemplace a la delgadez, lo cual se ve demostrado en que el grupo de bajo peso solamente tuvo un paciente y la media de IMC se ubique en sobrepeso. Al analizar los grupos en cuanto a las características demográficas no parecen tener diferencias en edad o género, aunque llama la atención que hubo un mayor número de pacientes con diabetes en el grupo de obesidad y obesidad mórbida, lo cual es esperable, pero desafortunadamente la cantidad de pacientes por grupo de IMC fue pequeña, lo que no permitió un análisis estadístico asociado a diabetes entre estos grupos.

Se dividió la población en dos grupos considerando un IMC de $30 \mathrm{~kg} / \mathrm{m}^{2}$, ya que los grupos de obesidad y obesidad mórbida fueron los que presentaron mayores diferencias. En la mayoría de los estudios, se utiliza 25 $\mathrm{kg} / \mathrm{m}^{2}$ como punto de corte para considerar obesidad o peso normal; sin embargo, en nuestro grupo de pacientes no hubo diferencias entre grupo con menor o mayor IMC utilizando $25 \mathrm{~kg} / \mathrm{m}^{2}$. Cuando se utilizó el punto de corte de 30, se encontraron diferencias. Entre las características previas no se encontraron discrepancias más que en la presencia de diabetes mellitus en el grupo de obesidad, lo cual, aunque sí se puede considerar un factor de riesgo para complicaciones, es algo esperado ya que la obesidad es factor de riesgo para desarrollo de ésta.

Las características, al ingreso, no mostraron diferencia en los puntajes de gravedad (SOFA y APACHE II); la mayor parte de los diagnósticos de ingreso fueron similares en ambos grupos, pero llama la atención que la presencia de sepsis fue mayor en el grupo de no obesos. Se ha reportado menor número de infecciones a nivel respiratorio y por causa fúngica en pacientes obesos, pero no se había reportado diferencia en sepsis de origen no pulmonar como sucedió en nuestros pacientes. ${ }^{5}$ En cuanto a los motivos de inicio de ventilación mecánica invasiva, la hipoxemia y la falla cardiaca fueron más comunes en el grupo de obesidad, esto debido probablemente a la fisiopatología de la obesidad per se y la interacción cardiopulmonar, con disminución de la capacidad funcional residual, colapso de vía aérea y formación de atelectasias, lo que favorece la hipoxe- mia. Estos cambios facilitan desarrollo de cor pulmonale, que junto con los cambios que genera la obesidad a nivel cardiaco explica que se presente mayor cantidad de falla cardiaca en este grupo de pacientes.

Respecto a la ventilación mecánica invasiva, se observa que, de manera inicial, tanto la oxigenación como la distensibilidad pulmonar fueron menores en el grupo de pacientes obesos, y aunque no fue significativa estadísticamente, se obtuvo una diferencia de $14 \%$ respecto al grupo de los no obesos. Esta diferencia es esperada por el efecto de la obesidad a nivel torácico que genera restricción en la pared del tórax, además del efecto de la obesidad abdominal que genera aumento de la presión intraabdominal y desplazamiento del diafragma hacia el tórax, todo esto produce disminución de la distensibilidad, principalmente de la pared torácica. Para corroborar lo anterior, necesitaríamos tener por separado el cálculo de la distensibilidad pulmonar, de la pared torácica y del sistema respiratorio. La disminución en la distensibilidad de los pacientes obesos, en comparación con los no obesos que se observó en nuestro estudio, es menor a la reportada previamente en otros estudios y corresponde a $35 \% ;^{7}$ esto puede deberse a que tales valores se obtuvieron en pacientes sanos, mientras que los valores de distensibilidad en nuestros pacientes se encuentran disminuidos en ambos grupos haciendo que la diferencia sea menor a la esperada.

Como se había comentado previamente, estas diferencias en la distensibilidad, con disminución de la capacidad funcional residual favorecen la disminución en la oxigenación y explican el que se requiera de mayor PEEP para el manejo de los pacientes obesos. En nuestros pacientes, se utilizó una media de $11 \mathrm{cmH}_{2} \mathrm{O}$ de PEEP en el grupo de pacientes obesos, lo cual está dentro de lo recomendado en la literatura y que corresponde a un PEEP mínimo entre 10 y 12 para evitar la formación de atelectasias, ${ }^{8}$ lo que evita hipoxemia. Esto se ve reflejado en la mejoría de la oxigenación que presentaron nuestros pacientes obesos, ya que posterior a las 48 horas con ventilación mecánica invasiva con PEEP, la diferencia en oxigenación ya no fue significativa. Se recomienda que en pacientes obesos se mantenga PEEP mínimo de 10 para prevenir el colapso y atelectasias, esto se ve reflejado en que a las 48 horas se continúe utilizando mayor PEEP en los pacientes obesos.

El volumen corriente fue mayor en el grupo de obesidad y esta diferencia fue significativa a las 48 horas. Al comparar el volumen corriente por peso predicho, llama la atención que se utilizaron volúmenes corrientes más altos, aunque esta diferencia es muy pequeña y es poco probable que tenga un impacto clínico. Esto nos explica las diferencias que se requieren para ventilar a los pacientes obesos. Esta diferencia puede ser porque se requiere mayor volumen corriente para mantener la 
ventilación normal en ellos; otra posible causa es que, a pesar de que nos basamos en la talla para el cálculo del volumen corriente adecuado, se tiende a sobrestimar las necesidades de volumen en estos pacientes y esto, a su vez, puede provocar que se calculen cifras mayores de volumen corriente en este grupo. Aun así, los valores se mantuvieron en el rango de protección alveolar.

Algunos estudios han reportado aumento en los días de ventilación mecánica en los pacientes con obesidad. En nuestro estudio no se observó este incremento y por lo contrario, fue mayor la duración de la ventilación mecánica en los pacientes no obesos que en los obesos, aunque esta diferencia no fue estadísticamente significativa. El subgrupo de pacientes con sobrepeso fue el que presentó mayor número de días con ventilación mecánica y estos datos son contrarios a lo reportado. Probablemente, esta diferencia se deba a los diagnósticos de ingreso de cada subgrupo, que si bien es cierto que el único diagnóstico con diferencia estadísticamente significativa fue diabetes mellitus, hubo más casos neurológicos o en estado postparo en el grupo de no obesos, los cuales se asocian con mayor tiempo para el retiro de la ventilación mecánica invasiva, independientemente del estado respiratorio.

La estancia en terapia intensiva fue ligeramente mayor en el grupo de pacientes obesos con diferencia solamente de dos días, sin presentar significancia estadística; en cuanto a los días de estancia intrahospitalaria, también fue mayor en pacientes obesos, con diferencia de una semana, sin que esto sea estadísticamente significativo. No se realizaron estudios de gastos económicos, pero una diferencia de una semana puede suponer un costo mucho más alto para los pacientes obesos en UTI al compararlos con los no obesos, lo cual deberá analizarse en un estudio posterior. Al observar los grupos por IMC, se encuentra una gran diferencia de estancia intrahospitalaria en el grupo de obesidad mórbida, pero con gran desviación estándar, esto debido a un caso que requirió de una estancia de 124 días para rehabilitación, lo que provocó una desviación en la curva con incremento secundario en el promedio de días de estancia intrahospitalaria en este grupo. A pesar de que no se encontró diferencia estadísticamente significativa, es de esperar una mayor estancia intrahospitalaria en el grupo de obesidad ya que suelen requerir mayor rehabilitación. Con estos pacientes es más difícil iniciar la movilización y se corren más riesgos de presentar complicaciones.

El uso de traqueostomía fue mayor en el grupo de no obesos, sin que esta diferencia fuera estadísticamente significativa. Esto correlaciona con el mayor tiempo de ventilación mecánica invasiva en dicho grupo y corresponde también al subgrupo de sobrepeso, igual que como correspondió en el caso de los días con ventilación.
En cuanto a la posición prono, ambos grupos fueron muy similares, aunque se pudiera pensar que si en el paciente obeso la oxigenación fue menor, se habría utilizado más esta maniobra; sin embargo, no fue necesaria la utilización de posición prono ya que los pacientes del grupo con obesidad mejoraron la oxigenación de forma satisfactoria a las 48 horas, correspondiendo de igual manera a causas de origen extrapulmonar, más que a causas pulmonares.

En cuanto a mortalidad, no se encontró diferencia estadísticamente significativa, con tan sólo $11 \%$, siendo mayor en el grupo de obesidad (40\%), cifra muy elevada que llama la atención ya que contrasta con lo que en la literatura se determina como «paradoja» de la obesidad, según la cual esperaríamos una menor mortalidad en estos pacientes. Al valorar la mortalidad según los subgrupos de IMC, observamos que el subgrupo con menor mortalidad fue el de sobrepeso y el de mayor el de obesidad mórbida; esto sí concuerda con lo reportado en la literatura donde el comportamiento de la mortalidad sigue una forma de $\mathrm{U}$; es decir, es mayor la mortalidad en los extremos. Hay que considerar que estos grupos son pequeños para compararlos, y entre grupos con el punto de corte de IMC de $30 \mathrm{~kg} / \mathrm{m}^{2}$, no se encontró diferencia estadística. Deberán hacerse estudios con mayor número de pacientes para poder corroborar dicha distribución en nuestra población de pacientes en terapia intensiva.

\section{CONCLUSIONES}

La obesidad es un padecimiento muy común en nuestro país, la cual se encuentra en aumento y se presenta de manera frecuente en los pacientes de terapia intensiva que requieren de ventilación mecánica invasiva. La literatura muestra una aparente mejoría de la mortalidad en los obesos, a pesar de que esperaríamos que presentaran mayor morbilidad y mortalidad al requerir ventilación mecánica invasiva. En este estudio se encontró aumento de mortalidad, pero no estadísticamente significativa. Se requerirá aumentar el número de pacientes para valorar si esta diferencia es real y determinar cómo afectan los diferentes parámetros de ventilación al pronóstico.

Se encontró diferencia significativa en los parámetros gasométricos con mayor afección de oxigenación al ingreso y en los parámetros de ventilación ya que requirieron mayor PEEP y mayor volumen corriente; esto se explica directamente por la fisiología pulmonar de los pacientes obesos.

No se encontraron diferencias significativas en días de ventilación mecánica y días de estancia en UCl. En el caso de estancia intrahospitalaria, la diferencia fue de una semana, pero con una desviación estándar amplia, probablemente por estancia prolongada de un pacien- 
te, por lo que no fue estadísticamente significativa. Se requerirá mayor número de pacientes para ver si esta diferencia se debe a esta estancia ya que una semana a pesar de no tener diferencia estadística, generaría un impacto clínico y económico. Del mismo modo, tampoco se logró encontrar una diferencia en el uso de posición prono y la necesidad de colocar traqueostomía.

Hay que considerar las diferencias en la fisiología de los pacientes obesos para la asistencia de ventilación mecánica invasiva. Necesitamos estudiar un número mayor de pacientes para poder valorar la mortalidad en nuestra población.

\section{BIBLIOGRAFÍA}

1. Pi-Sunyer FX. The obesity epidemic: pathophysiology and consequences of obesity. Obes Res. 2002;10 Suppl 2:97S-104S.

2. Kopelman PG. Obesity as a medical problem. Nature. 2000;404(6778):635-643.

3. Hernández AM, Shamah LT, Gómez AL, Gaona PE, GómezHumaran MM, Saturno HP, Villalpando HS. Encuesta Nacional de Salud y Nutrición de Medio Camino 2016 (ENSANUT2016). Instituto Nacional de Salud Pública; 2016.

4. O'Brien JM, Philips GS, Ali NA, Aberegg SK, Marsh CB, Lemeshow $S$. The association between body mass index, processes of care, and outcomes from mechanical ventilation: a prospective cohort study. Crit Care Med. 2012;40(5):1456-1463.

5. Wacharasint P, Boyd JH, Russell JA, Walley KR. One size does not fit all in severe infection: obesity alters outcome, susceptibility, treatment, and inflammatory response. Crit Care. 2013;17(3):R122.
6. Akinnusi ME, Pineda LA, EI Solh AA. Effect of obesity on intensive care morbidity and mortality: a meta-analysis. Crit Care Med. 2008;36(1):151-158.

7. Leme Silva P, Pelosi P, Rocco PR. Mechanical ventilation in obese patients. Minerva Anestesiol. 2012;78(10):1136-1145.

8. De Jong A, Chanques G, Jaber S. Mechanical ventilation in obese ICU patients: from intubation to extubation. Crit Care. 2017;21(1):63.

9. Anzueto A, Frutos-Vivar F, Esteban A, Bensalami N, Marks D, Raymondos K, et al. Influence of body mass index on outcome of the mechanically ventilated patients. Thorax. 2011;66(1):66-73.

10. Zhi G, Xin W, Ying W, Guohong X, Shuying L. "Obesity Paradox" in acute respiratory distress syndrome: asystematic review and meta-analysis. PLoS One. 2016;11(9):e0163677.

Patrocinios, relación de conflicto de intereses: El autor principal declara que no tiene ninguna inclinación comercial o asociativa que presente un conflicto de intereses con el trabajo presentado.

Correspondencia:

Alejandro Montaño Jiménez

Sur 136 Núm. 116 ,

Col. Las Américas,

Del. Álvaro Obregón, 01120,

Ciudad de México, México.

Tel: 5552308000 , ext. 8594

Cel: 5524988877

E-mail: dr.alejandromontano@gmail.com 